\title{
Local Decay of Solutions for Symmetric Hyperbolic Systems with Dissipative and Coercive Boundary Conditions in Exterior Domains
}

\author{
By \\ Nobuhisa IWASAKI
}

\section{\$. Introduction}

P. D. Lax and R. S. Phillips approached the scattering theory for wave equation or symmetric hyperbolic systems with conservative and coercive boundary conditions by time-depended methods in their book "Scattering Theory". We have seen whether the methods in this book are applicable to other cases or not. If the interest is restricted to local decay, they are applicable to solutions for symmetric hyperbolic systems with dissipative and coercive boundary conditions when the dimension of space-like $\geq 3$. We report this here.

We consider the following mixed problem in $\Omega \times[0, \infty)$

$$
\left\{\begin{array}{l}
\frac{\partial}{\partial t} u(x, t)=A u(x, t) \equiv \sum_{i=1}^{n} A_{i}(x) \frac{\partial}{\partial x_{i}} u(x, t)+C(x) u(x, t) \\
\left.B(x) u(x, t)\right|_{x \in \partial \Omega}=0 \\
u(x, 0)=f(x)
\end{array}\right.
$$

where $\Omega$ is an exterior domain with compact $C^{2}$-boundary in $R^{n}(n \geq 3), A$ is a symmetric dissipative and elliptic first order differential operator on $\Omega, A$ is of constant coefficient and $C(x) \equiv 0$ out of a bounded set of $\Omega$, moreover, $A$ holds the unique continuation theorem and $B(x)$ is dissipative and coercive with respect to $A$. Then, the solution $u(x, t)$ of $(0.1)$ is given by the contraction semigroup $U(t)$ on $\mathcal{L}^{2}(\Omega)$ like $u(x, t)=U(t) f$ if $f$ is in $\mathcal{L}^{2}(\Omega)$. We 
shall prove that

$$
\lim _{t \rightarrow \infty} U(t) f=0 \quad \text { in } \quad \mathcal{L}_{\text {loc }}^{2}(\bar{\Omega})
$$

except for static solutions though the operator norm of $U(t)$ on $\mathcal{L}^{2}(\Omega)=1$. This property is called local decay or decay principle. This is deduced from the followings;

1) $A$ has no point spectrum on imaginary axis except for origin.

2) The set of functions in $t:\{U(t+s) f, 0 \leq s<\infty\}$ is precompact in the space of uniformly bounded continuous $\mathcal{L}_{\text {loc }}^{2}(\bar{\Omega})$-valued functions.

1) follows from Lemma 4 in $\S 2.3,2$ ) is Lemma 5 in $\$ 2.3$ and we can deal abstractly with the proof of local decay from 1) and 2). We show this in $\S 1$ which is an extension of Appendix 2 of P. D. Lax and R. S. Phillips [9] to the case of contraction semigroup. The main theorem of this paper is Theorem 8.

T. Ikebe [4], M. Matsumura [12], K. Mochizuki [16], [17], G. Schmidt [21] and C. Wilcox [22] are the works concerned with existence of wave operator, local decay, limiting amplitude and eigenfunction expansions for symmetric (hyperbolic) system (general or special). There are many works of these problems for wave equation or Schrödinger operator. For example, we can give [2], $[3],[5],[6],[7],[9],[10],[13],[14],[15],[18],[19],[20]$.

\section{$\S 1$. Contraction Semi-group and Local Decay}

1.1. We begin by extending the classical result for one parameter group of unitary operators.

Let $X$ and $X^{\prime}$ stand for a reflexive Banach space and its dual space, respectively. Let $U(t)$ be a strong continuous one-parameter semi-group of contraction operators on $X$ and $A$ be the infinitesimal generator of $U(t)$. Moreover, let $\mathscr{B}_{[0, \omega)}[F]$ stand for the space of $F$-valued functions, which are continuous and bounded, having the uniform convergence topology on $[0, \infty)$, where $F$ is a closed subset of some locally convex topological vector space. Particularly, when $F=\boldsymbol{C}$, we denote it by $\mathcal{B}_{[0, \infty)}\left(=\mathcal{B}_{[0, \infty)}[C]\right)$.

We define the subset $N_{f}(u)$ of $\mathscr{B}_{[0, \infty)}$ by 


$$
N_{f}(u)=\left\{\varphi_{s}(t) ;[0, \infty) \ni t \rightarrow\langle U(t+s) u, f\rangle \in C ; s \in[0, \infty)\right\},
$$

where $u \in X$ and $f \in X^{\prime}$.

Theorem 1. We assume that $A$ has no point spectrum at $i \omega \in \boldsymbol{C}$ for any real number $\omega$, and that $N_{f}(u)$ is a pre-compact set of $\mathscr{B}_{[0, \infty)}$. Then,

$$
\lim _{t \rightarrow \infty} \varphi_{0}(t)=0 ; \quad \varphi_{0}(t) \equiv\langle U(t) u, f\rangle .
$$

Proof. Let us put $\lim _{t \rightarrow \infty} \sup \left|\varphi_{0}(t)\right|=\alpha$. We can choose a sequence $\left\{t_{i}\right\}_{i=1,2, \ldots}$, which diverges to infinity as $i \rightarrow \infty$, such that $\lim _{i \rightarrow \infty}\left|\varphi_{0}\left(t_{i}\right)\right|=\alpha$ and $\left\{\varphi_{t_{i}}(t)\right\}$ is a Cauchy sequence in $\mathscr{B}_{[0, \infty)}$, because $\bar{N}_{f}(u)$ is a compact set of $\mathscr{B}_{[0, \infty)}$. Let the continuous function $\Phi(t)$ $\in \mathcal{B}_{[0, \infty)}$ stand for the limit function of $\left\{\varphi_{t_{i}}(t)\right\}$. Then,

(1.1.1): $\Phi(t)$ can be extended to an almost periodic function on $(-\infty,+\infty)$.

Because, we can find a subsequence $\left\{s_{i}\right\}$ in an arbitrary sequence of real numbers $\left\{r_{j}\right\}$ which diverge to infinity such that $\left\{\Phi\left(t+s_{i}\right)\right\}$ is a uniformly convergent sequence of functions in $t$ in the following sense, that is,

(1.1.2): for any positive number $\varepsilon$ there exists a positive integer $N$ such that, if $j \geq i \geq N$, then

$$
\sup _{-s_{i} \leq t<\infty}\left|\Phi\left(t+s_{j}\right)-\Phi\left(t+s_{i}\right)\right|<\varepsilon, \quad\left(s_{i} \leq s_{j}\right) .
$$

In fact, if we take a proper subsequence $\left\{s_{i}\right\}$ of $\left\{r_{i}\right\},\left\{\varphi_{s_{i}}(t)\right\}$ is a Cauchy sequence in $\mathcal{B}_{[0, \infty)}$ and $s_{i} \leq s_{j}$ for $i<j$. And we may take a subsequence $\left\{t_{i}^{\prime}\right\}$ of $\left\{t_{i}\right\}$ such that $s_{i} \leq t_{i}^{\prime}$. On the other hand it holds that

$$
\begin{aligned}
\mid \Phi\left(t+s_{j}\right) & -\Phi\left(t+s_{i}\right)|\leq| \varphi_{t_{i}^{\prime}}\left(t+s_{j}\right)-\Phi\left(t+s_{j}\right) \mid \\
& +\left|\varphi_{t_{t^{\prime}}}\left(t+s_{i}\right)-\Phi\left(t+s_{i}\right)\right|+\left|\varphi_{s_{j}}\left(t+t_{i}^{\prime}\right)-\varphi_{s_{i}}\left(t+t_{i}^{\prime}\right)\right| .
\end{aligned}
$$

Here, if $j>i \geq N$ for some large integer $N$, the first and second terms of the right hand side are bounded by $\varepsilon / 3$ uniformly on $t+s_{i} \geq 0$ by the definition of $\Phi(t)$ and the third term is bounded by $\varepsilon / 3$ uniformly on $t+t_{i}^{\prime} \geq 0$, that is, on $t+s_{i} \geq 0$ by the way of choice of $\left\{s_{i}\right\}$. This means (1.1.2). 
From the property (1.1.2) we can conclude that $\Phi(t)$ is an almost periodic function in $t$ on $[0, \infty)$, that is, if we set

$$
m(\varepsilon) \equiv\{s \geq 0,|\Phi(t+s)-\Phi(t)|<\varepsilon\},
$$

$m(\varepsilon)$ is relatively dense in $[0, \infty)$. Since almost periodic function on $[0, \infty)$ can be extended uniquely to the almost periodic functions on $(-\infty,+\infty)$, we have (1.1.1).

Now, using that the generator $A$ has no point spectrum on the imaginary axis, we prove that $\Phi(t) \equiv 0$. From the mean ergodic theorem there exists $v_{\omega} \in X$ which is the limit vector of

$$
\frac{1}{T} \int_{0}^{T} e^{-i \omega t} U(t) u d t
$$

as $T \rightarrow \infty$ in the strong topology and which satisfies that

$$
e^{-i \omega t} U(t) v_{\omega}=v_{\omega},
$$

that is,

$$
v_{\omega} \in D(A) \text { and } A v_{\omega}=i \omega v_{\omega} .
$$

Hence $v_{\omega}$ must be zero vector. From the above all Fourier coefficients of the almost periodic function $\Phi(t)$, (i.e.)

$$
\lim _{T \rightarrow \infty} \frac{1}{T} \int_{0}^{T} e^{-i \omega t} \Phi(t) d t=\left\langle\lim _{T \rightarrow \infty} \frac{1}{T} \int_{0}^{T} e^{-i \omega t} U(t) u d t, f\right\rangle
$$

are zero. Hence we conclude that $\Phi(t) \equiv 0$ from the uniqueness theorem of almost periodic functions for their Fourier coefficients. By the construction of $\Phi(t)$ this shows that

$$
\alpha=\lim _{t \rightarrow \infty}\left|\varphi_{0}(t)\right|=\Phi(0)=0 .
$$

The proof is complete.

Remark. In the above proof of Theorem 1 we have used "nonexistence of point spectrum of $A$ on imaginary axis" only to prove that

$$
\lim _{T \rightarrow \infty} \frac{1}{T} \int_{0}^{T}\left\langle e^{-i \omega t} U(t) u, f\right\rangle d t=0 .
$$

Hence we have the following corollary.

Corollary 1. $\overline{N_{f}(u)}$ is a compact set of $\mathscr{B}_{[0, \infty)}$ and (1.1.3) holds 
for any real $\omega$, then $\lim _{t \rightarrow \infty} \varphi_{0}(t)=0 ; \varphi_{0}(t) \equiv\langle U(t) u, f\rangle$.

Let

$$
\beta_{g}(f)=\lim _{t \rightarrow \infty} \inf |\langle U(t) f, g\rangle|, \quad f \in X, g \in X^{\prime} .
$$

Theorem 2. The following i) and ii) are equivalent.

i) The generator $A$ has no point spectrum on the imaginary axis.

ii) For any $f \in X$ and $g \in X^{\prime}, \beta_{g}(f)$ is zero.

Proof. It is easy to show that ii) implies i). In fact, if $A$ has $i \omega$ as a point spectrum, there exist $f \in X$ and $g \in X^{\prime}$ such that $\langle f, g\rangle \neq 0$ and $U(t) f=e^{i \omega t} f$. This shows $\beta_{g}(f)=|\langle f, g\rangle| \neq 0$, in the other words, contradicts to the proposition ii).

Let us prove that i) implies ii). We fix $f$ an element of $X$. Then, the set $\{U(t) f, 0 \leq t<\infty\}$ is included in the subspace of $X$ which has a countable base because $U(t) f$ is continuous in $t$ in the strong topology of $X$. Hence we assume that $X$ has a countable base in the proof of this theorem. Let $G=\left\{g_{n}\right\}_{n=1,2, \ldots}$ be a countable base of $X^{\prime}$ and $G_{n}$ be the unit sphere of the finite dimensional subspace of $X^{\prime}$ generated by $\left\{g_{1}, \cdots, g_{n}\right\}$. And put

and

$$
\delta_{n}(f)=\lim _{t \rightarrow \infty} \inf \left\{\sup _{g \in \tilde{G}_{n}}|\langle U(t) f, g\rangle|\right\}
$$

$$
\delta(f)=\sup _{n=1,2, \cdots} \quad \delta_{n}(f)=\lim _{n \rightarrow \infty} \delta_{n}(f) .
$$

From the definition of $\delta(f)$ there exists the increasing sequences of real numbers $\left\{t_{i}\right\}$ and of integers $\left\{n_{i}\right\}$ which satisfy that

$$
\lim _{i \rightarrow \infty}\left[\sup _{g \in G_{n_{i}}}\left|\left\langle U\left(t_{i}\right) f, g\right\rangle\right|\right]=\delta(f),
$$

where $\lim _{i \rightarrow \infty} t_{i}=\lim _{i \rightarrow \infty} n_{i}=\infty$, and $\left\|U\left(t_{i}\right) f\right\| \leq\|f\|$ because $U(t)$ is a contraction semi-group. From the compactness of bounded sets in the weak topology of $X$, we can find the subsequence $\left\{t_{i}^{\prime}\right\}$ of $\left\{t_{i}\right\}$ such that the sequence of vectors $\left\{U\left(t_{i}^{\prime}\right) f\right\}$ converge to an element $\varphi$ of $X$ in the weak sense. We denote such a subsequence by $\left\{t_{i}\right\}$ again. Let us prove that

$$
\delta(\varphi) \geq \delta(f)
$$

If we fix $t \in[0, \infty)$, there exists $I(n, \varepsilon, t)$ for $\varepsilon>0$ such that 


$$
\sup _{g \in \sigma_{n}}|\langle U(t) \varphi, g\rangle| \geq \sup _{g \in G_{n}}\left|\left\langle U\left(t+t_{i}\right) f, g\right\rangle\right|-\varepsilon \quad i \geq I(n, \varepsilon, t),
$$

because $U(t)$ is a continuous operator in weak topology and $G_{n}$ is finite dimensional. If $i \rightarrow \infty$, this shows that

$$
\sup _{g \in \sigma_{n}}|\langle U(t) \varphi, g\rangle| \geq \delta_{n}(f)-\varepsilon .
$$

Since $t$ and $\varepsilon$ are arbitrary, $\delta_{n}(\varphi) \geq \delta_{n}(f)$. We have that

$$
\delta(\varphi) \geq \delta(f) .
$$

Next we prove that

$$
\|\varphi\| \leq \delta(f) .
$$

For any $\varepsilon>0$, there exists $N$ such that

$$
\|\varphi\| \leq \sup _{g \in G_{n}}|\langle\varphi, g\rangle|+\varepsilon,
$$

and there exists $I(N, \varepsilon)$ such that

$$
\begin{aligned}
\sup _{g \in G_{N}}|\langle\varphi, g\rangle| & \leq \sup _{g \in G_{N}}\left|\left\langle U\left(t_{i}\right) f, g\right\rangle\right|+\varepsilon, & & i \geq I(N, \varepsilon) \\
& \leq \sup _{g \in G_{n}}\left|\left\langle U\left(t_{i}\right) f, g\right\rangle\right|+\varepsilon, & & n \geq N,
\end{aligned}
$$

because $G_{n} \supset G_{N}$ when $n \geq N$.

From the way of choice of $\left\{t_{i}\right\}$ and $\left\{n_{i}\right\}$, if we put $n=n_{i}$ and make $i$ tend to infinity, the right hand side of the above inequality is replaced by $\delta(f)+\varepsilon$. Thus we have (1.1.5).

Since $\|\varphi\| \geq \delta(\varphi),(1.1 .4)$ and (1.1.5) mean that

$$
\|\varphi\|=\delta(\varphi)=\delta(f) .
$$

From this, we can prove that the subset $\Phi=\{U(t) \varphi, 0 \leq t<\infty\}$ is a precompact set of $X$ in the strong topology. In fact, $U(t) \varphi$ is continuous in $t$ in the strong topology and $\Phi$ is precompact in the weak topology. Therefore it is sufficient to prove that, if $\left\{U\left(t_{i}\right) \varphi\right\}$ is a convergent sequence in the weak topology, $\left\{U\left(t_{i}\right) \varphi\right\}$ is a convergent sequence in the strong topology, where $\left\{t_{i}\right\}$ is a sequence of real numbers which diverges to infinity. Let $\psi$ be the weak limit of $\left\{U\left(t_{i}\right) \varphi\right\}$, i.e.,

Then

$$
\psi=w-\lim _{i \rightarrow \infty} U\left(t_{i}\right) \varphi .
$$




$$
\begin{gathered}
\|\varphi\| \geq\left\|U\left(t_{i}\right) \varphi\right\| \geq\|\psi\|=\sup _{n} \sup _{g \in G_{n}}|\langle\psi, g\rangle| \\
\geq \delta(\varphi)=\|\varphi\| \quad \text { from }(1.1 .6) .
\end{gathered}
$$

Thus, we have that $\lim _{i \rightarrow \infty}\left\|U\left(t_{i}\right) \varphi\right\|=\|\psi\|$.

Applying the resonance theorem, we have the strong convergence of $\left\{U\left(t_{i}\right) \varphi\right\}$ to $\psi$. This means that $\Phi$ is precompact in the strong topology. Let $N(\varphi)$ stand for the set of all functions in $t: U(t+s) \varphi$, where $s$ denotes an arbitrary real non-negative constant. $N(\varphi)$ is the subset of $\mathscr{B}_{\mathrm{r} 0, \infty)}[X]$. Combining the compactness of $\Phi$ and the contractility of $U(t)$ (i.e.) $\|U(t)\| \leq 1$, we conclude that $N(\varphi)$ is a pre-compact subset of $\mathscr{B}_{[0, \infty)}[X]$. Particularly, for any $g \in X^{\prime} N_{g}(\varphi)$ is a pre-compact subset of $\mathscr{B}_{[0, \infty)}$. Applying Theorem $1,\langle\varphi, g\rangle$ must be zero, that is, $\varphi$ must be zero vector. This is nothing but $\delta(f)=0$ because $\delta(f)=\|\varphi\|$. In the other words, it holds the property ii) because $\bigcup_{n=1}^{\infty} G_{n}$ generates $X^{\prime}$. The proof is complete.

\subsection{To bring the previous result into a handier form for applications.}

Let $H$ be Hilbert space and $F$ be Fréchet space where $H$ is densely injected into $F$. We denote the inner product of $H$ by (,) and the norm of $H$ by $\|\cdot\|$. Moreover, we assume that the following "Condition $\mathbf{A}$ " is satisfied between $H$ and $F$.

Condition A. There exists a countable set of bilinear forms $\left\{\rho_{j}: j=1,2, \cdots\right\}$ on $F$ satisfying the following conditions (1) and (2).

(1) $\left\{\rho_{j}(f) \equiv\left[\rho_{j}(f, f)\right]^{1 / 2}, j=1,2, \cdots\right\}$ are semi-norms of $F$.

(2) for any $f \in H$, it holds that

$$
\rho_{j}(f) \leq \rho_{j+1}(f) \leq\|f\|, \sup _{j} \rho_{j}(f)=\|f\| \text {. }
$$

Remark 1. From the Condition $A$ it follows immediately that, setting $\sigma_{j}(f, g) \equiv(f, g)-\rho_{j}(f, g)$ for $f, g \in H, \sigma_{j}(f, g)$ are non-negative bilinear forms on $H$ and that $\sigma_{j}(f) \equiv\left[\sigma_{j}(f, f)\right]^{1 / 2}$ have the seminorms property, i.e.,

$$
\sigma_{j}(a f+b g) \leq|a| \sigma_{j}(f)+|b| \sigma_{j}(g) .
$$

Remark 2. If we set $H=L^{2}(\bar{\Omega})$ and $F=L_{\text {loc }}^{2}(\bar{\Omega})$, this pair satisfies the Condition A. 
Let $U(t)$ be a contraction semi-group on $H(t \geq 0)$, i.e., $\|U(t)\| \leq 1$, $A$ be the infinitesimal generator of $U(t), D(A)$ be the definition domain of $A$ and $M(f)$ be the motion of $f$, i.e.,

$$
M(f) \equiv U(\cdot) f \in \mathscr{B}_{[0, \infty)}[H] .
$$

Condition B. The set of motions $\{M(U(s) f)$, for $0 \leq s<\infty\}$ is precompact in $\mathscr{B}_{[0, \infty)}[F]$.

Condition C. The subset $\{U(t) f, 0 \leq t<\infty\}$ of $H$ is precompact in $F$.

Remark 3. Condition B implies Condition C.

Remark 4. If the set $\{f \in D(A) ;\|f\|+\|A f\| \leq 1\}$ is precompact in $F$, then all elements of $H$ satisfy the Condition C.

Let $K$ be the closed subset $\{f ;\|f\| \leq 1\} \subset H$. We introduce the following symbols.

$K_{S}$ stands for the set $K$ whose topology is equivalent to the strong topology of $H$.

$K_{F}$ stands for the set $K$ whose topology is equivalent to the topology of $F$.

$K_{W}$ stands for the set $K$ whose topology is equivalent to the weak topology of $H$.

Then, $K_{S}, K_{F}$ and $K_{W}$ are complete in each topology. The topology of $K_{S}$ is stronger than the topology of $K_{F}$ and the topology of $K_{F}$ is stronger than the topology of $K_{W}$, i.e.,

$$
K_{S} \subset K_{F} \subset K_{W} \cdot
$$

It is clear from the Condition A that $K_{S} \subset K_{F}$. Using (2) of the Condition A and Remark 1,

$$
\begin{aligned}
|(f, g)| & \leq\left|\sigma_{j}(f, g)\right|+\left|\rho_{j}(f, g)\right| \\
& \leq \sigma_{j}(f) \sigma_{j}(g)+\rho_{j}(f) \rho_{j}(g) \\
& \leq\|f\| \sigma_{j}(g)+\rho_{j}(f)\|g\| .
\end{aligned}
$$

This means that a Cauchy sequence of $K_{F}$ is a Cauchy sequence of $K_{W}$ because for a fixed $g \in H \lim _{j \rightarrow \infty} \sigma_{j}(g)=0$. Thus $K_{F} \subset K_{W}$, and since it is clear that $K_{S}$ and $K_{W}$ are complete, $K_{F}$ is also complete. From (1.2.1) we conclude that 


$$
\mathscr{B}_{[0, \infty)}\left[K_{S}\right] \subset \mathscr{B}_{[0, \infty)}\left[K_{F}\right] \subset \mathscr{B}_{[0, \infty)}\left[K_{W}\right] \text {. }
$$

Remark 5. If $T$ is a bounded linear operator on $H, T$ is continuous in the weak topology of $H$. Hence, if $T$ is a contraction operator, $T$ is a closed operator on $K_{F}$. Particularly, if $T$ is restricted on a subset $E$ of $K_{F}$ whose image by $T, T E$, is precompact in $K_{F}, T$ is continuous on $\bar{E}$ (in $K_{F}$ ).

Theorem 3. A has no point spectrum on imaginary axis if and only if it holds the proposition that, if (and only if) $f \in H$ satisfies the Condition $B$,

$$
\lim _{t \rightarrow \infty} \rho_{j}(U(t) f)=0 \quad \text { for all } j .
$$

Proof. We may assume that $\|f\| \leq 1$. Then, $U(t) \in \mathscr{B}_{[0, \infty)}\left[K_{s}\right]$, and the Condition B means that $\{M(U(s) f) \quad 0 \leq s<\infty\}$ is precompact in $\mathscr{B}_{[0, \infty)}\left[K_{F}\right]$, that is, in $\mathscr{B}_{[0, \infty)}\left[K_{W}\right]$ from (1.2.2). Therefore for a fixed $g \in H, \quad N_{g}(f)=\left\{\varphi_{s}(t) \equiv(U(t+s) f, g) \in \mathscr{B}_{[0, \infty)} \quad 0 \leq s<\infty\right\}$ is precompact in $\mathcal{B}_{[0, \infty)}$.

Applying Theorem 1,

$$
\lim _{t \rightarrow \infty} \varphi_{0}(t)=0 \text {. }
$$

Now, we put $\delta_{j}=\lim _{t \rightarrow \infty} \sup \rho_{j}(U(t) f)$. Then, there exists the Cauchy sequence $\left\{U\left(t_{k}\right) f\right\}$ in $K_{F}$ such that $\delta_{j}=\lim _{k \rightarrow \infty} \rho_{j}\left(U\left(t_{k}\right) f\right)$ and $\lim _{k \rightarrow \infty} t_{k}=\infty$ from Remark 3. If we denote the limit vector of $\left\{U\left(t_{k}\right)\right\}$ as $k \rightarrow \infty$ by $h$, then $\delta_{j}=\rho_{j}(h)$ and $\lim _{k \rightarrow \infty} \psi\left(t_{k}\right)=\|h\|^{2}$ where $\psi(t) \equiv(U(t) f, h)$. On the other hand, $\lim _{t \rightarrow \infty} \psi(t)=0$ from (1.2.3). Thus $h$ must be zero vector, that is, $\delta_{j}=0$. The "if" part of this theorem is clear.

The proof is complete.

If we assume the precompactness of the set $\{U(t) f\}$ in $F$, we have the similar result as Theorem 2 . We put

$$
\varepsilon(f) \equiv \sup _{j}\left\{\liminf _{t \rightarrow \infty} \rho_{j}(U(t) f)\right\} .
$$

Theorem 4. A has no point spectrum on imaginary axis if and only if it holds the proposition that, if $f$ satisfies the Condition $C$, then $\varepsilon(f)$ must be zero.

Proof. We assume that $\|f\| \leq 1$. Considering the precompact- 
ness of the set $\{U(t) f\}$ in $F$, there exist sequences of real numbers $\left\{t_{i}\right\}$ and $\left\{p_{N}\right\}$ which diverge to infinity and satisfy that, for $n \geq N$,

$$
\left|\rho_{p_{N}}\left(U\left(t_{n}\right) f\right)-\varepsilon(f)\right|<\frac{1}{N},
$$

and that $\left\{U\left(t_{k}\right) f\right\}$ is a Cauchy sequence in $K_{F}$. Therefore, there exists $\Phi \in K_{F}$ such that $\Phi=\lim _{k \rightarrow \infty} U\left(t_{k}\right) f$ in $K_{F}$. Considering that $U(s)$ is continuous on $\bar{E} ; E=\left\{\lambda\left(U\left(s_{1}\right)-U\left(s_{2}\right)\right) f ;|\lambda| \leqq 1 / 2,0 \leqq s_{1}, s_{2} \leqq \infty\right\}$ $\subset K_{F}$ (Remark 5) and $\left(U\left(t_{k}\right)-\Phi\right) / 2 \in \bar{E}$, when we fix $\varepsilon>0, s$ and $p$, we can take $q$ and constant $C_{\varepsilon s p}$ such that

Thus

$$
\rho_{p}\left(U\left(t_{k}+s\right) f-U(s) \Phi\right) \leq C_{\varepsilon s p} \rho_{q}\left(U\left(t_{k}\right) f-\Phi\right)+\varepsilon .
$$

$$
\rho_{p}(U(s) \Phi) \geq \rho_{p}\left(U\left(t_{k}+s\right) f\right)-C_{\varepsilon s p} \rho_{q}\left(U\left(t_{k}\right) f-\Phi\right)-\varepsilon .
$$

If we make $k$ tend to infinity, the second term converges to zero. Since $\varepsilon$ is arbitrary, we have that

$$
\begin{gathered}
\rho_{p}(U(s) \Phi) \geq \liminf _{\substack{t \rightarrow \infty \\
\varepsilon(\Phi) \geq \varepsilon(f) .}} \rho_{p}(U(t) f), \quad \text { that is, }
\end{gathered}
$$

On the other hand, $\|\Phi\|=\lim _{p \rightarrow \infty} \rho_{p}(\Phi)=\varepsilon(f)$ from (1.2.4). This implies

$$
\begin{gathered}
\varepsilon(\Phi) \geq \varepsilon(f)=\|\Phi\| \geq \lim _{t \rightarrow \infty}\|U(t) \Phi\| \geq \varepsilon(\Phi), \quad \text { that is, } \\
\varepsilon(\Phi)=\|\Phi\|=\|U(t) \Phi\| .
\end{gathered}
$$

Since $\{U(t) \Phi\}$ is included in the closure of $\{U(t) f\}$ in $K_{F},\{U(t) \Phi\}$ is precompact in $K_{F}$, too. We now prove that $\Phi$ satisfies the condition B. To do so, it is enough to show that

(1.2.6): if the sequence $\left\{U\left(t_{i}\right) \Phi\right\}$ is a Cauchy sequence in $K_{F}$ and $\left\{t_{i}\right\}$ tends to infinity as $i \rightarrow \infty$, then it is a Cauchy sequence in $K_{S}$.

Because, $U(t)$ is a contraction semi-group and strong continuous in $t$. From (1.2.5), for $\varepsilon>0$ there exists $N$ and $T$ such that for $n \geq N$ and $t \geq T$

Hence

$$
\sigma_{n}(U(t) \Phi)=\|U(t) \Phi\|-\rho_{n}(U(t) \Phi)<\varepsilon .
$$

$$
\left\|U\left(t_{i}\right) \Phi-U\left(t_{j}\right) \Phi\right\|
$$




$$
\begin{aligned}
& \leq \sigma_{n}\left(U\left(t_{i}\right) \Phi-U\left(t_{j}\right) \Phi\right)+\rho_{n}\left(U\left(t_{i}\right) \Phi-U\left(t_{j}\right) \Phi\right) \\
& \leq \sigma_{n}\left(U\left(t_{i}\right) \Phi\right)+\sigma_{n}\left(U\left(t_{j}\right) \Phi\right)+\rho_{n}\left(U\left(t_{i}\right) \Phi-U\left(t_{j}\right) \Phi\right) \\
& \leq \varepsilon+\varepsilon+\varepsilon,
\end{aligned}
$$

if we make $i$ and $j$ be sufficiently large. This shows (1.2.6), that is, $\Phi$ satisfies the Condition B. Hence, we can use theorem 3 for $\Phi$. We conclude that $\Phi$ must be zero vector, that is, $\varepsilon(f)=0$. The "if" part of this theorem is clear. The proof is complete.

We know of the contraction semi-group that there exists the strong limite of $\frac{1}{T} \int_{0}^{T} e^{-i \omega t} U(t) u d t$ as $T \rightarrow \infty$ and except for countable real numbers

$$
\lim _{T \rightarrow \infty} \frac{1}{T} \int_{0}^{T} e^{-i \omega t} U(t) u d t=0 .
$$

We now denote by $\left(\omega_{i}, a_{i}\right)$ the countable pairs of real number and vector such that

$$
\lim _{T \rightarrow \infty} \frac{1}{T} \int_{0}^{T} e^{-i \omega_{i} t} U(t) u d t=a_{i} \neq 0 .
$$

Moreover, we have that

$$
\sum_{j=1}^{\infty}\left\|a_{j}\right\|^{2} \leq \lim _{T \rightarrow \infty} \frac{1}{T} \int_{0}^{T}\|U(t) u\|^{2} d t, \quad\left(A-i \omega_{j}\right) a_{j}=0 ; a_{j} \in D(A)
$$

and

$$
U(t)\left(\sum_{j=1}^{\infty} a_{j}\right)=\sum_{j=1}^{\infty} e^{i \omega_{j} t} a_{j} .
$$

Hence, if we put $P u=\sum_{j=1}^{\infty} a_{j}, P$ is a projection on $H$, i.e. $P^{2}=P$ and the range of $P$ coincides with the closure of the subspace consisted of eigenvectors whose eigenvalues are on imaginary axis.

On the other hand, in the proof of Theorem 3, we can replace the assumption that $A$ has no point spectrum on the imaginary axis by the assumption that for any real $\omega$

$$
\lim _{T \rightarrow \infty} \frac{1}{T} \int_{0}^{T} e^{-i \omega t} U(t) u d t=0
$$

Therefore, applying Theorem 3 to $f-P f$, we have the following Theorem.

Theorem 5. If $f$ satisfies the Condition $B$, then

$$
\lim _{t \rightarrow \infty} \rho_{j}(U(t) f-U(t) P f)=0 \quad \text { for all } j .
$$

Here $U(t) P f$ is nothing but a H-valued almost periodic functions in $t$. 
Corollary 5. If $\{U(t) f\}$ is precompact in $H$, then

$$
\lim _{t \rightarrow \infty}\|U(t) f-U(t) P f\|=0 \text {. }
$$

\section{§2. Applications to Solutions for Symmetric Hyperbolic Systems with Dissipative and Coercive Boundary Conditions}

\subsection{Existence and regularity of solutions.}

We consider the mixed initial-boundary value problem for hyperbolic system of the form :

$$
\left\{\begin{aligned}
\frac{\partial}{\partial t} u(x, t) & =\sum_{i=1}^{n} A_{i}(x) \frac{\partial}{\partial x_{i}} u(x, t)+C(x) u(x, t) & ;(x, t) \in \Omega \times[0, \infty) \\
u(x, t) & \in B(x) & ;(x, t) \in \partial \Omega \times[0, \infty) \\
u(x, 0) & =u_{0}(x) & ; x \in \Omega
\end{aligned}\right.
$$

in the product space of a $n$-dimensional domain $\Omega$ with compact $C^{2}$-boundary and $[0, \infty)$. Here, we assume that $A$ and $B$ satisfy the following conditions where

$$
A=\sum_{i=1}^{n} A_{i}(x) \frac{\partial}{\partial x_{i}}+C(x) .
$$

Condition I. $A$ is a formally dissipative operator, that is,

i) $A_{i}$ are $k \times k$ Hermitian-symmetric-matrix-valued functions of class $\mathscr{B}^{1} \subset C^{1}$.

ii) $C+C^{*}-\sum_{j=1}^{n} \frac{\partial}{\partial x_{j}} A_{j} \leq 0, \quad x \in \Omega$.

Condition II. $A$ is a uniformly elliptic operator, that is, $\left|\sum_{j=1}^{n} A_{j} \xi_{j}\right| \geq \delta|\xi|, \delta>0$ for any non-zero real vector $\left(\xi_{j}\right)$, where $\delta$ is independent on $x \in \Omega$.

Condition III. $B(x)$ are maximal non-positive, that is, the matrix $A_{n}(x)$ is non-positive over $B(x)$, i.e.,

$$
u A_{n} u \leq 0
$$

for all $u(x)$ in $B(x), x \in \partial \Omega$, and $B(x)$ are the maximal subspace in $C^{k}$ satisfying such a property, where $A_{n}(x)=\sum_{j}^{n} A_{j}(x) n_{j}(x)$ and $\left(n_{j}(x)\right)$ is the outer normal to the surface $\partial \Omega$ at $x \in \partial \Omega$. 
Condition IV. $B(x)$ are coercive with respect to $A$, that is, $B \cap \varepsilon(\xi)=\{0\}$, for any non-zero real vector $\left(\xi_{j}\right)$ such that $\sum_{j=1}^{n} \xi_{j} \eta_{j}=0$, where $\varepsilon(\xi)$ are the subspace spanned by the root-vectors of $M(\xi)=$ $A_{n}^{-1} \sum_{j=1}^{n} A_{j} \xi_{j}$ whose eigen-values have the negative real parts.

We now mention the results deduced by the above conditions. From Conditions II and IV, we have the coercive estimate for $u \in H^{1}(\Omega)$ and $\left.u\right|_{\partial \Omega} \in B$;

$$
\left\|\frac{\partial}{\partial x_{i}} u\right\| \leq \text { Const }\left\{\|A u\|+\| u_{\|}^{\prime} \mid\right\},
$$

where $H^{1}(\Omega)$ stands for the vector-valued square integrable functions with square integrable first derivatives. We denote by $D(\mathscr{A})$ the functions which are elements of $H^{1}(\Omega)$ and satisfy the boundary conditions (i.e.) $\left.u\right|_{\partial \Omega} \in B$. From Conditions I, II, III and IV, we have that the differential operator $(\lambda-A)$ is one to one and onto map from $D(\mathcal{A})$ to $\mathcal{L}^{2}(\Omega)$ for any positive number $\lambda$ and satisfies the estimate ;

$$
\|u\| \leq \frac{1}{\lambda}\|i(\lambda-A) u\| .
$$

(Refer to P. D. Lax and R. S. Phillips [8] and [9] for the precise discussions for these properties.)

Let $\mathcal{A}$ stand for the closed operator attached to $A$ on $\mathcal{L}^{2}(\Omega)$ with $D(\mathscr{A})$ as definition domain. Then, the following lemma holds from the above fact applying the Hille-Yosida's Theorem.

Lemma 1. $\mathcal{A}$ is an infinitesimal generator of a contraction semi-group.

We denote by $U(t)$ the contraction semi-group generated by $\mathcal{A}$. Then, it holds for $f \in D(\mathcal{A})$ that

$$
\frac{d}{d t} U(t) f=\mathscr{A} U(t) f \quad \text { and } \quad U(t) f \in D(\mathscr{A}) .
$$

This equation means the mixed initial-boundary value problem (2.1.1) and $U(t) f$ is its solution.

\subsection{The case in a bounded domain.}

In this section 2.2 , we assume that the domain $\Omega$ is a bounded 
domain. Using the coerciveness of $\mathcal{A}$, Rellich's Theorem and Remark 4 in 1.2 , we have that $\{U(t) f\}$ is precompact in $\mathcal{L}^{2}(\Omega)$. Considering Corollary 5, we immediately have the following conclusion.

Theorem 6. If (2.1.1) satisfies Conditions I, II, III and IV and $\Omega$ is a bounded domain, then there exists the projection $P$ on $\mathcal{L}^{2}(\Omega)$ such that:

i) $U(t) f$ is a unique solution of (2.1.1) with the initial data $f$.

ii) $U(t) P f$ is an almost periodic solution of (2.1.1).

iii) $\lim _{t \rightarrow \infty}\|U(t) f-U(t) P f\|_{\mathcal{L}^{2}(\Omega)}=0$.

Corollary 6. We assume that $A+i \mu$ is a system holding the unique continuation theorem ( $\mu$ is real) and that (2.1.1) satisfies Condition I, II, III and IV. Moreover, we assume the stronger Condition $V$. Then any solution $U(t) f$ of (2.1.1) decays as $t \rightarrow \infty$ in $\mathcal{L}^{2}(\Omega)$, i.e.,

$$
\lim _{t \rightarrow \infty}\|U(t) f\|_{\mathcal{L}^{2}(\Omega)}=0 .
$$

Here the Condition $\mathrm{V}$ is the followings;

Condition V. $(A, B)$ is strictly dissipative, that is, the following i) or ii) holds :

i) If $u \in B$ and $\int_{\partial \Omega} u \cdot A_{n} u d S=0$, then there exists an open set $\omega$ of $\partial \Omega$ such that $u \equiv 0$ on $\omega$.

ii) There exists a point $x_{0}$ of $\bar{\Omega}$ such that $C+C^{*}-\sum_{i=1}^{n} \frac{\partial}{\partial x_{j}} A_{j}$ is a negative matrix at $x_{0}$.

Proof of Corollary. Since the range of $P$ coincides with the subspace spanned by eigenfunctions of $\mathcal{A}$ on $\mathcal{L}^{2}(\Omega)$ whose eigenvalues lie on imaginary axis, it is enough to prove the following lemma.

Lemma 2. We assume that $A-i \mu$ holds the unique continuation theorem and satisfies Conditions I, III and V. If $(A-i \mu) u=0, u \in$ $D(\mathcal{A})$ and $\mu$ is real, then $u$ must be zero.

Remark. In this lemma it is irrespective for $\Omega$ to be bounded or unbounded.

Proof. It holds that 


$$
\begin{aligned}
0 & =\operatorname{Re}((A-i \mu) u, u) \\
& =\frac{1}{2}\left(\left(A+A^{*}\right) u, u\right)+\operatorname{Re} \int_{\partial \Omega} u \cdot A_{n} u d S,
\end{aligned}
$$

where $A^{*}$ is the formal adjoint operator of $A$. Since $A+A^{*}$ is nonpositive matrix and $\int_{\partial \Omega} u \cdot A_{n} u d S$ is non-positive, too, $\left(\left(A+A^{*}\right) u, u\right)$ and $\int_{\partial \Omega} u \cdot A_{n} u d S$ must be zero. Thus, there exists an open set $\omega_{0}$ in $\partial \Omega$ or an open set $\omega_{1}$ in $\Omega$ such that $u \equiv 0$ on $\omega_{0}$ or $u \equiv 0$ on $\omega_{1}$ from Condition V. Using the unique continuation theorem we conclude $u \equiv 0$ on $\Omega$. The Lemma is proved.

\section{3. The case in an exterior domain.}

In this section 2.3 we assume that $A$ is a constant coefficient differential operator without term $C$ at the exterior of some bounded domain, that is, the form

$$
A=\sum_{j=1}^{n} A_{j} \frac{\partial}{\partial x_{j}} .
$$

(Remark. we call this condition Condition VI.) and that the dimension of $x$-space is not less than 3 and $\Omega$ is an exterior domain.

2.3.1. Solutions of systems of constant coefficients in free space. We consider the equation of the form

$$
\left\{\begin{array}{l}
\frac{\partial}{\partial t} u=A u \\
\left.u\right|_{t=0}=f,
\end{array}\right.
$$

where $A$ is of constant coefficients in whole space $\boldsymbol{R}^{n}$. Since $A$ defines the self-adjoint operator $\mathscr{A}_{0}$ on $\mathcal{L}^{2}$ with $H^{1}$ as its definition domain, we can express by the strong continuous one-parameter group of unitary operator of $\mathcal{L}^{2}: U_{0}(t)$ the unique solution of the initial value problem for (2.3.1) with initial data $f$ like $U_{0}(t) f$.

We denote the orthonormal eigenvectors and their eigenvalues of symmetric matrices:

$$
A(\xi)=\sum_{j=1}^{n} A_{j} \xi_{j}
$$

with parameter $\xi$ of real vectors by $\gamma_{i}(\xi)$ and $\tau_{i}(\xi), i=1, \cdots, k$, respectively, where 


$$
\begin{aligned}
& \tau_{1}(\xi) \geq \tau_{2}(\xi) \geq \cdots \geq \tau_{k / 2}(\xi)>0, \\
& \gamma_{k / 2+j}(\xi)=\gamma_{k / 2-j+1}(-\xi) \text { and } \tau_{k / 2+j}(\xi)=-\tau_{k / 2-j+1}(-\xi) .
\end{aligned}
$$

We denote by $N$ the subset of $\mathcal{L}^{2}\left(S_{n-1}\right)$ consisting of the functions which have the form:

$$
\sum_{i=1}^{k / 2} n_{i}(\omega) \gamma_{i}(\omega), \quad \omega \in S_{n-1},
$$

where $\mathcal{L}^{2}\left(S_{n-1}\right)$ is the space of square integrable functions valued in $\boldsymbol{C}^{k}$ on $S_{n-1}$ and $h_{i}(\omega)$ are scalar-valued functions. Let $f(x)$ be an element of $\mathcal{L}^{2}, \hat{f}(\xi)=\mathscr{F}(f)$ be Fourier Transform of $f$ and $\phi_{j}(\sigma, \omega)$ be the scalar products of $\hat{f}(\sigma \omega)$ and $\gamma_{j}(\omega)$;

$$
\phi_{j}(\sigma, \omega)=\hat{f}(\sigma \omega) \cdot \gamma_{j}(\omega), \quad j=1, \cdots, k,
$$

where we introduce polar coordinates $\xi=\sigma \omega, \sigma \in \boldsymbol{R}^{1}$ and $\omega \in S_{n-1}$. Then we can define the operator $\Phi$ from $\mathcal{L}^{2}=\mathcal{L}^{2}\left(\boldsymbol{R}^{n}\right)$ to $L^{2}\left(\boldsymbol{R}^{1}, N\right)$, which is the space of $N$-valued square integrable functions on $\boldsymbol{R}^{1}$, such that

$$
\phi(f) \equiv \sigma^{\left[\frac{n-1}{2}\right]}|\sigma|^{\frac{n-1}{2}-\left[\frac{n-1}{2}\right]}\left(\sum_{j=1}^{k / 2} \phi_{j}(\sigma, \omega) \gamma_{j}(\omega)\right)
$$

and $\Phi$ is unitary map. In fact,

$$
\begin{aligned}
\|f\|_{\mathcal{L}^{2}}^{2} & =\|\hat{f}\|_{\mathcal{L}^{2}}^{2} \\
& =\sum_{j=1}^{k} \int_{S_{n-1}} d \omega \int_{0}^{+\infty}|\sigma|^{n-1}\left|\phi_{j}(\sigma, \omega)\right|^{2} d \sigma \\
& =\sum_{j=1}^{k / 2} \int_{S_{n-1}} d \omega \int_{-\infty}^{+\infty}|\sigma|^{n-1}\left|\phi_{j}(\sigma, \omega)\right|^{2} d \sigma \\
& =\int_{S_{n-1}} \int_{-\infty}^{+\infty}|\phi(f)|^{2} d \omega d \sigma,
\end{aligned}
$$

because $\phi_{j}(\sigma, \omega)=\phi_{k-j+1}(-\sigma,-\omega)$. Conversely, if we correspond $\psi(\sigma, \omega) \in L^{2}\left(\boldsymbol{R}^{1}, N\right)$ to $g(x)$ such that

$$
\begin{gathered}
g(x) \equiv \overline{\mathscr{F}}(\tilde{\psi}(\xi)) ; \\
\tilde{\psi}(\xi)=|\xi|^{\left[\frac{n-1}{2}\right]-\frac{n-1}{2}}\left\{|\xi|^{\left[\frac{n-1}{2}\right]} \psi\left(|\xi|, \frac{\xi}{|\xi|}\right)+(-|\xi|)^{\left[\frac{[-1}{2}\right]} \psi\left(-|\xi|,-\frac{\xi}{|\xi|}\right)\right\} .
\end{gathered}
$$

Then, $g(x)$ belongs to $\mathcal{L}^{2}$ and $\psi(\sigma, \omega)=\Phi(g)$. Moreover, to make clear the properties of solutions of (2.3.1), we define the operator $\mathscr{I}_{n}$ from functions in $(s, \omega)$ to functions in $x$ by the form: 


$$
\mathscr{I}_{n}(h) \equiv C_{n} \int_{S_{n-1}}\left(\frac{1}{i} \frac{\partial}{\partial s}\right)^{\left[\frac{n-1}{2}\right]} h(x \omega, \omega) d \omega
$$

where $h(s, \omega)$ is an element of $C^{\infty}\left(\boldsymbol{R}^{1}, N\right)$ which is the set of $N$ valued $C^{\infty}$-functions in $s \in \boldsymbol{R}^{1}$. Then it holds the following properties for $\mathscr{I}_{n}:$ if $h \in C_{0}^{\infty}\left(\boldsymbol{R}^{1}, N\right)$

$$
\begin{aligned}
& \mathscr{L}_{n}(h)= \begin{cases}\phi^{-1}(\hat{h}(\sigma, \omega)), & \text { when } n \text { is odd, } \\
\phi^{-1}\left(|\sigma|^{-1 / 2} \hat{h}(\sigma, \omega)\right), & \text { when } n \text { is even; }\end{cases} \\
& \hat{h}(\sigma, \omega)=\frac{1}{\sqrt{2 \pi}} \int_{-\infty}^{+\infty} e^{i \sigma s} h(s, \omega) d s=\overline{\mathscr{I}}_{(s)}(h(s, \omega))
\end{aligned}
$$

and

$$
\left(f, \mathscr{I}_{n}\left[\left(\frac{1}{i} \frac{\partial}{\partial s}\right)^{\left[\frac{n}{2}\right]-\left[\frac{n-1}{2}\right]} h\right]\right)_{x}=\left(\mathscr{F}_{(\sigma)}(\phi(f)), K h\right)_{(s, \omega)},
$$

where

$$
(K h)(s, \omega)=\left\{\begin{array}{l}
h(s, \omega), \quad \text { when } n \text { is odd } \\
\mathscr{F}_{(\sigma)}\left(\sigma|\sigma|^{-1 / 2} \overline{\mathscr{F}}_{(s)}(h)\right)(s, \omega), \quad \text { when } n \text { is even. }
\end{array}\right.
$$

Moreover, if $J \Phi(f) \in L^{2}\left(\boldsymbol{R}^{1}, N\right)$ and $\mathscr{F}_{(\sigma)}(J \Phi(f)) \in C^{\infty}(|s|<R, N)$, then

$$
f(x)=\mathscr{I}_{n}\left(\mathscr{F}_{(\sigma)}(J \Phi(f)), \text { in }|x|<R,\right.
$$

where $J \Phi(f)= \begin{cases}\Phi(f), & \text { when } n \text { is odd } \\ |\sigma|^{+1 / 2} \Phi(f), & \text { when } n \text { is even. }\end{cases}$

We now define the closed subspace $D_{-}$of $\mathcal{L}^{2}$ by the closure of functions which are represented as the form:

$$
f(x)=\mathscr{I}_{n}\left\{\left(\frac{1}{i} \frac{\partial}{\partial s}\right)^{\left[\frac{n}{2}\right]-\left[\frac{n-1}{2}\right]} h(s, \omega)\right\}
$$

where $h(s, \omega)$ is an element of $C_{0}^{\infty}\left(\boldsymbol{R}^{1}, N\right)$ whose support is contained in $\boldsymbol{R}_{-}^{1}=(-\infty, 0]$. Then we have the property that, if $f \in D_{-}$, $U_{0}(t) f=0$ at $|x|<-t \cdot C_{\min }, C_{\min }=\min _{1 \leq i \leq k / 2} \inf _{|\xi|=1} \tau_{i}(\xi)$, because, if $f(x)$ is represented as (2.3.5), $U_{0}(t) f$ is represented as the form:

$$
\begin{aligned}
& U_{0}(t) f=C_{n} \sum_{j=1}^{k / 2} \int_{|\omega|=1} \tilde{h}_{j}\left(x \omega-\tau_{j}(\omega) t, \omega\right) \gamma_{j}(\omega) d \omega ; \\
& \tilde{h}_{j}(s)=\left(\frac{1}{i} \frac{\partial}{\partial s}\right)^{\left[\frac{n}{2}\right]} h(s, \omega) \cdot \gamma_{j}(\omega),
\end{aligned}
$$

and that, if $f \in D_{-}^{\perp}$, from (2.3.3), 


$$
\begin{cases}\mathscr{F}_{(\sigma)}(\Phi(f))(s, \omega)=0, & \text { at } s<0, \text { when } n \text { is odd, } \\ \mathscr{F}_{(\sigma)}\left(\sigma|\sigma|^{-1 / 2} \Phi(f)\right)=0, & \text { at } s<0, \text { when } n \text { is even. }\end{cases}
$$

Therefore, when $n$ is odd, if $f \in D^{\perp}$, then

$$
U_{0}(t) f=0 \text { at } \quad|x|<C_{\min } t .
$$

When $n$ is even, if $f \in D^{\perp} \cap H^{1 / 2}$, from (2.3.5)

$$
\mathscr{F}_{(\sigma)}\left(|\sigma|^{1 / 2} \Phi(f)\right)=\alpha \int_{0}^{\infty} \frac{1}{s-\delta} \mathscr{F}_{(\sigma)}\left(\sigma|\sigma|^{-1 / 2} \Phi(f)\right)(s, \omega) d s \quad \text { at } \quad s<0,
$$

then

$$
\begin{aligned}
& U_{0}(t) f=C_{n} \sum_{j} \int g_{j}\left(x \omega-\tau_{j}(\omega) t, w\right) \gamma_{j}(\omega) d \omega \quad \text { at } \quad|x|<C_{\min } t ; \\
& g_{j}(s, \omega)=\alpha_{n} \int_{0}^{\infty} \frac{1}{(s-\delta)^{[(n-1) / 2]+1}} h_{j}(\delta, \omega) d s, \quad s>0 \\
& h_{j}(s, \omega)=\mathscr{L}_{(\sigma)}\left(\sigma|\sigma|^{-1 / 2} \Phi(f)\right) \cdot \gamma_{j}(\omega) .
\end{aligned}
$$

Applying the Schwartz's inequality to this expression, we have

$$
\begin{aligned}
& \left|U_{0}(t) f\right|^{2} \leq \beta_{n} \frac{\|f\|_{1 / 2}^{2}}{\left(C_{\min } t-|x|\right)^{n-1}}, \quad \text { at } \quad C_{\min } t>|x| ; \\
& \|f\|_{1 / 2}^{2}=\left\|(|\xi|+)^{1 / 2} \hat{f}(\xi)\right\|_{\mathcal{L}^{2}}^{2} .
\end{aligned}
$$

Putting the above in order, we have

\section{Lemma 3.}

1) If $f \in D_{-}, U_{0}(t) f=0$ at $|x|>-C_{\min } t$.

2) When $n$ is odd, if $f \in D_{-}^{\perp}$,

$$
U_{0}(t) f=0 \text { at } \quad|x|<+C_{\min } t .
$$

When $n$ is even, if $f \in D^{\perp} \cap H^{1 / 2}$,

$$
\left|U_{0}(t) f\right|^{2} \leq \beta_{n} \frac{\|f\|_{1 / 2}^{2}}{\left(C_{\min } t-|x|\right)^{n-1}}, \quad \text { at } \quad|x|<C_{\mathrm{min}} t .
$$

Theorem 7. If $f=0$, at $|x|>\rho$, when $n=$ odd,$\quad U_{0}(t) f=0 \quad$ at $\quad|x|<C_{\min }(t-\rho)$, when $n=$ even, $\quad\left|U_{0}(t) f\right|^{2} \leq \rho_{n} \frac{\|f\|_{1 / 2}^{2}}{\left(C_{\min }(t-\rho)-|x|\right)^{n-1}}$ at $|x|<C_{\min }(t-\rho)$.

Proof. This is clear because we can show that $U_{0}(\rho) f$ belongs 
to $D^{\perp}$ and $\|f\|_{1 / 2}=\left\|U_{0}(t) f\right\|_{1 / 2}$ by Fourier Transform.

Next, we show a lemma which means that any operator attached to $A$ on $\mathcal{L}^{2}$-space in exterior domain has no point spectrum on imaginary axis except for zero.

Lemma 4. If $u$ is square-integrable in $|x|>R$ and satisfies the equation ;

$$
(A+i \mu) u=0 \quad \text { in } \quad|x|>R
$$

where $\mu$ is a non-zero real number, then $u$ must be zero in $|x|>R$.

Remark. In this Lemma it is not necessary for $A$ to be nonsingular (i.e.) elliptic. It is sufficient to assume that $A$ is symmetric.

This lemma has been shown in P. D. Lax and R. S. Phillips [9] Chapter 6 when the dimension of $x$-space $n$ is odd. This is proved in the same way when it is even, too. In order to prove simply we use the result of D. Ludwig [11] on the Radon Transform.

We define $\tilde{R}_{0}$ operator from $\mathscr{D}_{L^{2}}$ to $\mathscr{D}_{L^{2}}\left(L^{2}\left(S_{n-1}\right)\right)$ by the form:

$$
\begin{aligned}
\left(\mathcal{R}_{0} f\right)(s) & =\mathscr{F}_{(\sigma)}\left(\sigma^{n-1} h(\sigma, \bullet)\right): \\
h(\sigma, \omega) & =\mathscr{I}_{(x)}(f)(\sigma \cdot \omega), \quad \sigma \in \boldsymbol{R}^{1}, \omega \in S_{n-1}
\end{aligned}
$$

Lemma (Ludwig). $\tilde{\mathscr{R}}_{0}$ is a one-to-one, onto, bicontinuous operator from $\mathscr{D}_{L^{2}}$ to $\tilde{\mathscr{R}}_{0}\left(\mathscr{D}_{L^{2}}\right) \subset \mathscr{D}_{L^{2}}\left(L^{2}\left(S^{n-1}\right)\right)$ and from $\mathscr{D}$ to $\mathscr{D}\left(L^{2}\left(S^{n-1}\right)\right) \cap \tilde{N}$, and $u=0$ in $|x|>R$ if and only if $\tilde{R}_{0} u=0$ in $|s|>R$, where we define that $f \in \mathscr{D}\left(L^{2}\left(S^{n-1}\right)\right)$ is contained in $\tilde{N}$ if $f$ is an even function in $(s, \omega)$ and satisfies that

$$
\int_{-\infty}^{\infty} \int_{S_{n-1}} s^{k} f(s, \omega)_{l} Y(\omega) d s d \omega=0
$$

for all spherical harmonic $Y_{l}(\omega)$ of degree $l$ when $k<l+(n-1)$.

Where $\mathscr{D}_{L^{2}}$ is the space of functions of $L^{2}$ in $x \in \boldsymbol{R}^{n}$, every derivative of which belongs to $L^{2}$ and $\mathscr{D}_{L^{2}}\left(L^{2}\left(S^{n-1}\right)\right)$ is the space of $L^{2}\left(S_{n-1}\right)$-valued functions in $s \in \boldsymbol{R}^{1}$ satisfying this property. $\mathscr{D}$ is compact support $C^{\infty}$-functions and $\mathscr{D}\left(L^{2}\left(S_{n-1}\right)\right)$ is $L^{2}\left(S^{n-1}\right)$-valued $\mathscr{D}$ functions.

Remark. $\tilde{R}_{0}$ which we define here is equal to $\left(\frac{\partial}{\partial s}\right)^{n-1} \mathscr{R}$ in D. Ludwig [11]. So we have a little modified the necessary and sufficient condition. 
Proof of Lemma 4. We may assume that $u$ belongs to $\mathscr{D}_{L^{2}}$ because we may put $u=0$ in $|x| \leq R$ and we can convolute the mollifier to $u$ since the differential operator is of constant coefficients. The operator $\tilde{\mathcal{R}}_{0}$ has the properties that

$$
\tilde{\mathcal{R}}_{0}\left(\frac{\partial}{\partial x_{i}} f\right)=\omega_{i} \frac{\partial}{\partial s} \tilde{\mathcal{R}}_{0}(f) .
$$

Therefore, $\tilde{u}=\tilde{\mathscr{R}}_{0}(u)$ satisfies the equation:

$$
\begin{aligned}
& \left(A(\omega) \frac{\partial}{\partial s}+i \mu\right) \tilde{u}(s, \omega)=0, \quad|s|>R, \\
& A(\omega)=\sum A_{i} \omega_{i},
\end{aligned}
$$

because $\left(A+i_{\mu}\right) u=0,|x|>R$. Since there exists $\widehat{A}(\omega)$ such that $\widehat{A}(\omega) A(\omega) A(\omega)=A(\omega)$, (2.3.6) implies that

$$
A(\omega) \tilde{u}( \pm s, \omega)=e^{ \pm(R-s) i \mu \tilde{A}(\omega)} \cdot A(\omega) \tilde{u}( \pm R, \omega) \text {, where } s>R .
$$

$\tilde{u}(s, \omega)$ belongs to $\mathscr{D}_{L^{2}}\left(L^{2}\left(S_{n-1}\right)\right) . \quad A(\omega) \tilde{u}(s, \omega)$, also, belongs to $\mathscr{D}_{L^{2}}\left(L^{2}\left(S_{n-1}\right)\right)$. Thus, $A(\omega) \tilde{u}(s, \omega)$ must be zero in $|s|>R$. From (2.3.6) $\tilde{u}(s, \omega)$ must be zero because $\mu \neq 0$. We have shown that $\tilde{u}(s, \omega)$ is in $\mathscr{D}\left(L^{2}\left(S_{n-1}\right)\right)$. If we put $\tilde{f}=\left(A(\omega) \frac{\partial}{\partial s}+i \mu\right) \tilde{u}$, then $\tilde{f}$ belongs to $\mathscr{D}\left(L^{2}\left(S_{n-1}\right)\right) \cap \tilde{N}$. Since $\mu \neq 0$, we can use the induction in $k$ to show that $\tilde{u}$ belongs to $\tilde{N}$, because

$$
\iint s^{k} \tilde{f} \cdot Y_{l} d s d \omega=\iint k s^{k-1} A(\omega) \tilde{u} \cdot Y_{l} d s d \omega+i \mu \iint s^{k} \tilde{u} \cdot Y_{l} d s d \omega
$$

where $v \cdot Y_{l}=\left(v_{1} Y, v_{2} Y_{l}, \cdots\right), v=\tilde{f}, \tilde{u}$ or $A(\omega) \tilde{u}$. It is clear from the definition that $\tilde{u}$ is even in $(s, \omega)$. Thus, we conclude that $u(x) \equiv 0$ in $|x|>R$ from the Lemma (Ludwig).

\subsubsection{The decay of the solution for mixed-boundary value prob-} lem in exterior domain.

Here, we take $\mathcal{L}^{2}(\bar{\Omega})$ as $H$ and $\mathcal{L}_{\text {loc }}^{2}(\bar{\Omega})$ as $F$ in 1.2 . If we assume Condition VI and that $\Omega$ is an exterior domain, we shall show that $U(t) f$ satisfies the Condition B using Lemma 3 and that $U(t) f$ decays locally applying Theorem 3 , because there is Lemma 4 , where $U(t) f$ is a contraction semi-group which corresponds the initial data $f$ to the function at $t$ fixed of solution for mixed problem 
(2.1.1) which we defined in Section 2.1, that is, which satisfies the Condition I, II, III and IV.

Lemma 5. If $U(t)$ is the above, $\Omega \subset R^{n}(n \geq 3)$ is an exterior domain and (2.1.1) satisfies Condition VI, then the set of motions $M(U(s) f)$ for $0 \leq s<\infty$ is precompact in $\mathscr{B}_{\mathrm{I0}, \infty)}\left(\mathcal{L}^{2}{ }_{\mathrm{loc}}(\bar{\Omega})\right)$, for every $f \in \mathcal{L}^{2}(\Omega)$, namely, $f$ satisfies Condition $B$ as $F=\mathcal{L}^{2}{ }_{\text {loc }}(\bar{\Omega})$.

Proof. Since there is the contractility of $U(t)$, it is sufficient to prove it only for $f$ 's which form a dense subset in $\mathcal{L}^{2}(\Omega)$. So we assume that $f$ is with compact support and belongs to the definition domain of $\mathcal{A}: \mathscr{D}(\mathcal{A})$, that is, belongs to $H^{1}(\bar{\Omega})$ and satisfies the boundary condition on $\partial \Omega$. So we have that

$$
\text { Const } \begin{aligned}
\|U(t) f\|_{H^{1}(\Omega)} & \leq\|A U(t) f\|_{\mathcal{L}^{2}(\Omega)}+\|U(t) f\|_{\mathcal{L}^{2}(\Omega)} \\
& \leq\|A f\|_{\mathcal{L}^{2}(\Omega)}+\|f\|_{\mathcal{L}^{2}(\Omega)} \cdot
\end{aligned}
$$

We now fix such a $f$. We may assume that the support of $f$ is contained in $\{|x|<\rho\} \cap \Omega$ where $\rho>\rho_{0}$ and $A$ is the constant coefficient differential operator discussed in 2.3.1 in $\left\{|x|>\rho_{0}\right\}$ which is contained in $\Omega$. Then, it is clear that $f$ is orthogonal to every element of $D_{-}^{-\rho_{1}}=U_{0}\left(-\rho_{1}\right) D_{\text {- }}$ when we suppose that $f$ is an element of $\mathcal{L}^{2}\left(\mathbb{R}^{n}\right)$ by putting $f \equiv 0$ in $\Omega^{c}$. Because $g=0$ in $\{|x| \leq \rho\}$ if $g \in D^{\rho_{1}}, \rho_{1}=\rho / C_{\min }$, from Lemma 3. Moreover, we can prove that $U(t) f$ have a same property, namely,

$$
U(t) f \text { belongs to }\left(D_{-}^{-\rho_{1}}\right) \text {. }
$$

In fact, we have the duality such that

$$
(U(t) f, g)_{\mathcal{L}^{2}(\Omega)}=\left(f, U_{0}(-t) g\right)_{\mathcal{L}^{2}\left(\mathbb{R}^{n}\right)}
$$

because, if $g \in D_{-}^{-\rho_{1}}$, then, $g \in \mathcal{L}^{2}(\Omega)$ and it holds that

$$
U_{0}(-t) g=U(t)^{*} g
$$

from uniqueness of the dual operator $U(t)^{*}$ which give solutions of the hyperbolic system with some dissipative boundary condition. Since $U_{0}(-t) g$ is an element of $D_{-}$, too,

$$
(U(t) f, g)_{\mathcal{L}^{2}(\Omega)}=\left(f, U_{0}(-t) g\right)_{\mathcal{L}^{2}\left(\boldsymbol{R}^{n}\right)}=0 .
$$

By using the Rellich's theorem it is clear from (2.3.7) that $\{U(t) f$, 
$0 \leq t<\infty\}$ is pre-compact in $\mathcal{L}^{2}{ }_{\text {loc }}(\bar{\Omega})$.

We shall prove the following inequality: If we give $\varepsilon>0$ and $\rho^{\prime}$, then there exists $\rho^{\prime \prime}$ such that for all $s, t$ and $\tau \geq 0$

$$
\begin{aligned}
& \|U(\tau+s) f-U(\tau+t) f\|_{\mathcal{L}^{2}\left(|x| \leq \rho^{\prime}\right)} \\
& \leq 3\|U(s) f-U(t) f\|_{\mathcal{L}^{2}\left(|x| \leq \rho^{\prime \prime}\right)}+\varepsilon .
\end{aligned}
$$

In order to show this we take a $C^{\infty}$-function $\chi(x)$ such that $|\chi(x)|$ $\leq 1, \chi(x) \equiv 1$ in $|x| \geq \rho$ and $\chi(x)=0$ in $\left\{|x| \leq \rho_{0}\right\}$ and we consider the following one.

$$
\begin{aligned}
& U(\tau+s) f-U(\tau+t) f \\
& =U(\tau)(1-\chi(x))\{U(s) f-U(t) f\} \\
& \quad+\left[U(\tau) \chi(x)\{U(s) f-U(t) f\}-\chi(x) U_{0}(\tau) \chi(x)\{U(s) f-U(t) f\}\right] \\
& \quad+\chi(x) U_{0}(\tau) \chi(x)\{U(s) f-U(t) f\} .
\end{aligned}
$$

$\mathcal{L}^{2}$-norm of the first term of the right hand side of (2.3.10) is bounded by $\|(1-\chi(x))\{U(s) f-U(t) f\}\|$. So, we have that for all $s, t$ and $\tau \geq 0$

$$
\begin{aligned}
& \|U(\tau)(1-\chi(x))\{U(s) f-U(t) f\}\|_{\mathcal{L}^{2}(\Omega)} \\
\leq & \|U(s) f-U(t) f\|_{\mathcal{L}^{2}(|x| \leq \rho)} .
\end{aligned}
$$

Since $U(s) f \in\left(D_{-}^{-\rho_{1}}\right)^{\perp}, \chi(x) U(s) f \in\left(D_{-}^{-\rho_{1}}\right)^{\perp}$. So $U_{0}\left(\rho_{1}\right) \chi(x) U(s) f \in\left(D_{-}\right)^{\perp}$. Thus, from Lemma 3 we have

$$
\begin{aligned}
& \left\|\chi(x) U_{0}(\tau) \chi(x) U(s) f\right\|_{\mathcal{L}^{2}\left(|x| \leq \rho^{\prime}\right)} \\
\leq & \left\|U_{0}(\tau) \chi(x) U(s) f\right\|_{\mathcal{L}^{2}\left(|x| \leq \rho^{\prime}\right)} \\
\leq & \left\{\begin{array}{rr}
0, & \text { when } n \text { is odd } \\
\rho_{n} \frac{\|\chi(x) U(s) f\|_{1 / 2}}{\left(C_{\min } \tau-\rho-\rho^{\prime}\right)^{(n-1) / 2}} \cdot \rho^{\prime n-1}, & \text { at } \quad \rho^{\prime}<C_{\min }\left(\tau-\rho_{1}\right), \\
\text { when } n \text { is even. }
\end{array}\right.
\end{aligned}
$$

Therefore, we have that, at $\tau>\rho^{\prime} / C_{\min }+\rho_{1}$,

$$
\begin{gathered}
\| \text { the third term } \|_{\mathcal{L}^{2}\left(|x|<\rho^{\prime}\right)} \\
\leq \begin{cases}0, & \text { when } n \text { is odd } \\
\frac{\text { Const }}{\left(\tau-\rho_{1}-\rho^{\prime} / C_{\min }\right)^{(n-1) / 2}} \cdot\|f\|_{1}, & \text { when } n \text { is even }\end{cases}
\end{gathered}
$$


because $\|\chi(x) U(s) f\|_{1 / 2} \leq$ Const $\|U(s) f\|_{1} \leq$ Const $\|f\|_{1}$, that is, there exists $\tau_{0}$ such that for $\tau \geq \tau_{0}$

$$
\left\|\chi(x) U_{0}(\tau) \chi(x)\{U(s) f-U(t) f\}\right\|_{\mathcal{L}^{2}\left(|x|<\rho^{\prime}\right)} \leq \varepsilon / 2 .
$$

Next we estimate the second term. We operate $\frac{\partial}{\partial t}-A$ to the second term $\equiv I_{2}(\tau)$. We have that

$$
\begin{aligned}
& \left(\frac{\partial}{\partial t}-A\right) I_{2}(\tau) \\
& \quad=A(\chi) \cdot U_{0}(\tau) \chi(x)\{U(s) f-U(t) f\} \equiv G(\tau): \\
& A(\chi)=\sum A_{i} \frac{\partial}{\partial x_{i}} \chi(x), \quad \text { in } \quad \Omega \times[0, \infty)
\end{aligned}
$$

and $I_{2}(\tau)$ belongs to $D(\mathcal{A})$. So that,

$$
I_{2}(\tau)=U\left(\tau-\tau_{1}\right) I_{2}\left(\tau_{1}\right)+\int_{\tau_{1}}^{\tau} U(\tau-\sigma) G(\sigma) d \sigma .
$$

This implies the estimate that

$$
\begin{aligned}
& \left\|I_{2}(\tau)\right\|_{\mathcal{L}^{2}\left(|x|<\rho^{\prime}\right)} \\
\leq & \left\|I_{2}(\tau)\right\| \mathcal{L}^{2}\left(R^{n}\right) \\
\leq & \mid I_{2}\left(\tau_{1}\right)\left\|_{\mathcal{L}^{2}\left(\boldsymbol{R}^{n}\right)}+\int_{\tau_{1}}^{\tau}\right\| G(\sigma) \|_{\mathcal{L}^{2}\left(\boldsymbol{R}^{n}\right)} d \sigma .
\end{aligned}
$$

Since $A(\chi) \equiv 0$ at $|x| \geq \rho$, we can take $\rho^{\prime}=\rho$ in (2.3.12) and we have that at $\sigma \geq 2 \rho / C_{\min }$

$$
\|G(\sigma)\|_{\mathcal{L}^{2}\left(\boldsymbol{R}^{n}\right)} \leq \begin{cases}0, & \text { when } n \text { is odd, } \\ \frac{\text { Const }}{\left(\sigma-2 \rho / C_{\min }\right)^{(n-1) / 2}}\|f\|, & \text { when } n \text { is even. }\end{cases}
$$

Since we here assume $n \geq 3$,

$$
\begin{aligned}
& \int_{\tau_{1}}^{\tau}\|G(\sigma)\|_{\mathcal{L}^{2}\left(\boldsymbol{R}^{n}\right)} d \sigma \leq\left\{\begin{array}{cc}
0, & n=\text { odd } \\
\text { Const }\|f\|_{1} \frac{1}{\left(\tau_{1}-2 \rho / C_{\text {min }}\right)^{(n-3) / 2}}, & n=\text { even }
\end{array}\right. \\
& \text { at } \tau, \tau_{1} \geq 2 \rho / C_{\text {min }} \text {. }
\end{aligned}
$$

This means that there exists $\tau_{1}$ such that for $\tau \geq \tau_{1}$

$$
\left\|I_{2}(\tau)\right\|_{\mathcal{L}^{2}\left(\boldsymbol{R}^{n}\right)} \leq\left\|I_{2}\left(\tau_{1}\right)\right\|_{\mathcal{L}^{2}\left(\boldsymbol{R}^{n}\right)}+\varepsilon / 2 .
$$

On the other hand, $U(t)$ has the finiteness of the dependence domain 
such that

$$
\|U(t) g\|_{\mathcal{L}^{2}\left(\left\{|x|>R+C_{\max } t\right\} \cap \Omega\right)} \leq\|g\|_{\mathcal{L}^{2}(\{|x|>R\} \cap \Omega)} .
$$

In $|x| \geq \rho, G(\sigma) \equiv 0$ and $I_{2}(0) \equiv \chi(x)\{1-\chi(x)\}\{U(s) f-U(t) f\} \equiv 0$. Therefore, we have that $I_{2}\left(\tau_{1}\right)=0$ in $|x| \geq \rho+\tau_{1}$, namely,

$$
\begin{aligned}
& \left\|I_{2}\left(\tau_{1}\right)\right\|_{\mathcal{L}^{2}\left(\boldsymbol{R}^{n}\right)}=\left\|I_{2}\left(\tau_{1}\right)\right\|_{\mathcal{L}^{2}\left(|x| \leq \rho+C_{\max } \tau_{1}\right)} \cdot \\
& \left\|I_{2}\left(\tau_{1}\right)\right\|_{\mathcal{L}^{2}\left(\boldsymbol{R}^{n}\right)} \leq 2|| U(s) f-U(t) f \|_{\mathcal{L}^{2}\left(|x| \leq \rho+2 C_{\max } \tau_{1}\right)} . \\
& \text { Because } \quad I\left(\tau_{1}\right)=U\left(\tau_{1}\right) \chi(x)\{U(s) f-U(t) f\} \\
& -\chi(x) U_{0}\left(\tau_{1}\right) \chi(x)\{U(s) f-U(t) f\} \text {, }
\end{aligned}
$$

and $U(t)$ and $U_{0}(t)$ have the finiteness of dependence domain such that

$$
\begin{aligned}
& \|U(t) g\|_{\mathcal{L}^{2}(\{|x|<R\} \cap \Omega)} \leq\|g\|_{\mathcal{L}^{2}\left(\left\{|x| \leq R+C_{\max } t\right\} \cap \Omega\right)} \\
& \left\|U_{0}(t) g\right\|_{\mathcal{L}^{2}(|x| \leq R)} \leq\|g\|_{\mathcal{L}^{2}\left(|x| \leq R+C_{\max } t\right)} .
\end{aligned}
$$

From the above estimates (2.3.11), (2.3.13), (2.3.14) and (2.3.15) we have that if $\tau \geq \max \left\{\tau_{0}, \tau_{1}\right\}$ and $\rho^{\prime \prime} \geq \rho+2 C_{\max } \tau_{1}$ it holds (2.3.9). Moreover, when $\tau \leq \max \left\{\tau_{0}, \tau_{1}\right\}=\tau_{2}$, if we use (2.3.16), we have that

$$
\begin{gathered}
\|U(\tau)(U(s) f-U(t) f)\|_{\mathcal{L}^{2}\left(|x| \leq \rho^{\prime}\right)} \\
\leq\|U(s) f-U(t) f\|_{\mathcal{L}^{2}\left(|x| \leq \rho^{\prime}+C_{\max } \tau_{2}\right)} .
\end{gathered}
$$

So, we conclude (2.3.9) for all $\tau \geq 0$.

We have defined the mapping $M$ from the subset $\mathcal{S} \equiv\{U(s) f$, $0 \leq s<\infty\}$ of $\mathcal{L}^{2}$ loc $(\bar{\Omega})$ to the subset of Motions $\{M(U(s) f), 0 \leq s<\infty\}$ of $\mathscr{B}_{[0, \infty)}\left(\mathcal{L}^{2}{ }_{\text {loc }}(\bar{\Omega})\right)$ by

$$
M(g) \equiv U(t) g, \quad g \in \mathcal{S}
$$

which is a $\mathcal{L}^{2}$ loc $(\bar{\Omega})$-valued function in $t$. Then the inequality (2.3.9) means that $M$ is extendable to a continuous mapping on $\bar{S} \subset \mathcal{L}^{2}{ }_{\text {loc }}(\bar{\Omega})$. Therefore, $\{M(U(s) f), 0 \leq s<\infty\}$ is precompact in $\mathcal{B}_{[0, \infty)}\left(\mathcal{L}_{\text {loc }}^{2}(\bar{\Omega})\right)$ because $\bar{S}$ is compact itself. The proof is complete.

Lemma 5 shows that Theorem 3 or Theorem 5 is applicable to the solution of (2.1.1) in the case of exterior domain, namely, we can conclude the following theorem. 
Let $U(t)$ be a semi-group such that $U(t) f$ represents the solution of (2.1.1).

Let $\Re$ be a subspace of $\mathcal{L}^{2}(\Omega)$ such that

$$
\begin{aligned}
\Re & =\text { the closure of Range } \mathscr{A} \\
& =\text { the closure of Range }(U(1)-I) \\
& =\left\{f ; \lim _{T \rightarrow \infty} \frac{1}{T} \int_{0}^{T} U(t) f d t=0\right\} .
\end{aligned}
$$

Theorem 8. If the mixed problem (2.1.1) in exterior domain contained in $\boldsymbol{R}^{n}(n \geq 3)$ satisfies Conditions $I, I I, I I I, I V$ and Condition $V I$ and if $A-i \mu$ holds the unique continuation theorem for real $\mu$, then the solution $U(t) f$ has a limit in $\mathcal{L}_{\text {loc }}^{2}(\bar{\Omega})$ as $t \rightarrow \infty$ and its limit $g$ is a static solution of (2.1.1.) belonging to $\mathcal{L}^{2}(\bar{\Omega})$, that is, $U(t) g=g$. Particularly, if $f \in \mathfrak{I}$, then the limit is zero. Moreover, if we assume Condition $V$, then, $\Omega$ coincides with $\mathcal{L}^{2}(\bar{\Omega})$.

Proof. From Lemma 5, $U(t)$ satisfies the condition of Theorem 5 if we take $H=\mathcal{L}^{2}(\Omega)$ and $F=\mathcal{L}_{\text {loc }}^{2}(\bar{\Omega})$. So, we have the projection $P$ on $\mathcal{L}^{2}(\Omega)$;

$$
\lim _{t \rightarrow \infty}\|U(t) f-U(t) P f\|_{\mathcal{L}^{2}(\{|x| \leq \rho\} \cap \Omega)}=0
$$

and considering Lemma 4 we have that the Range $P$ must coincide with $\Re$ because there is no eigenvalue of $\mathcal{A}$ on imaginary axis except for the origin. In fact, let $u \in \mathcal{L}^{2}(\bar{\Omega})$ be an eigenvector with eigenvalue $i_{\mu}$ of $\mathcal{A}$ where $\mu$ is real, the Lemma 4 shows that $u$ must be identically zero at $|x| \geq \rho_{0}$ for some real $\rho_{0}$. Since it holds the unique continuations theorem for $A-i \mu, u$ must be identically zero on $\Omega$. For the last part of Theorem from Lemma $2 i_{\mu}=0$ is not an exceptional point. This is that Range $P=\mathscr{L}=\mathcal{L}^{2}(\Omega)$. The Theorem is proved.

\section{References}

[1] BGir., H., Fastperiodische Funktion, Erg. Miath., Springer, Berlin, 1932.

[2] Eidus, D. M., On the principle of limiting absorption, Mat. Sb. 57 (1962), 13-44.

[3] Ikebe, T., Scattering for the Schrödinger operator in an exterior domain, J. Math. Kyoto Univ. 7 (1967), 93-112.

[47 . Scattering for uniformly propagative systems, Proc. International Conference on Functional Analysis and Related Topics (1969), to appear. 
[5] Iwasaki, N., On the principle of limiting amplitude, Publ. RIMS Kyoto Univ. Ser. A 3 (1968), 373-392.

[6] Kiyama, S., On the exponential decay of solutions for the wave equation with the potential function, Osaka J. Mat. 4 (1967), 15-35.

[7] Ladyzenskaja, O. A., On the principle of limiting amplitude, Usp. Mat. Nauk 12 (1957), 161-164.

[8] Lax, P. D. and R. S. Phillips, Local boundary conditions for dissipative symmetric linear differential operators, Comm. Pure Appl. Math. 13 (1960), 427-455.

[9] , Scattering Theory, Academic Press, New York, 1967.

[10] Lax, P. D., C. S. Morawetz and R. S. Phillips, The exponential decay of solution of the wave equation in the exterior of a star-shaped obstacle, Bull. Amer. Math. Soc. 68 (1962), 593-595.

[11] Ludwig, D., The Radon transform on Euclidean space, Comm. Pure Appl. Math. 19 (1966), 49-81.

[12] Matsumura, M., Comportement des solutions de quelques problèmes mixtes pour certains systèmes hyperboliques symétriques à coeffecients constants, Publ. RIMS Kyoto Univ. Ser. A 4 (1968), 309-359.

[13] Mizohata, S., Sur l'analyticité de la fonction spectrale de l'opérateur $\Delta$ relatif au problème extérieur, Proc. Japan Acad. 39 (1963), 352-357.

[14] , Theory of Partial Differential Equations, Iwanami, Tokyo, 1966 (Japanese).

[15] Mizohata, S. and K. Mochizuki, On the principle of limiting amplitude for dissipative wave equations, J. Math. Kyoto Univ. 6 (1966), 109-127.

[16] Mochizuki, K., Spectral theory and scattering for symmetric hyperbolic systems in an exterior domain, Publ. RIMS Kyoto Univ. 5, this issue.

[17] - The principle of limiting amplitude for hyperbolic systems in an exterior domain, Publ. RIMS Kyoto Univ. 5, this issue.

[18] Morawetz, C. S., The decay of solutions of the exterior initial-boundary value problem for the wave equation, Comm. Pure Appl. Math. 14 (1961), 561-569.

[19] - The limiting amplitude principle for arbitrary finite bodies, Comm. Pure Appl. Math. 18 (1965), 183-189.

[20] Shenk II, N. A., Eigenfunction expansions and scattering theory for the wave equation in an exterior region, Arch. Rational Meth. Anal. 21 (1966), 120-150,

[21] Schmidt, G., Spectral and scattering theory for Maxwell's equations, Arch. Rational Mech. Anal. 28 (1968), 283-322.

[22] Wilcox, C., Wave operators and asymptotic solutions of wave propagation problems of classical physics, Arch. Rational Mech. Anal. 22 (1966), 37-78.

[23] Yosida, K., Functional Analysis, Springer, Berlin, 1965. 